Cuad. Invest. Filol., 48 (2020), 85-95. http://doi.org/10.18172/cif.4520

\title{
FACETAS DE LA MELANCOLÍA EN LA NOVELA OTOÑO DE ALI SMITH
}

\author{
María Luisa Hernández García \\ Universidad Complutense de Madrid \\ mluisa.hernandezg@gmail.com
}

\begin{abstract}
RESUMEN: Ali Smith es una gran creadora de atmósferas melancólicas. En su novela Otoño nos sumerge en la añoranza de la vida por parte de un hombre centenario, Daniel; añoranza que toma tintes politicos en la joven Elisabeth, enfrentada a la pérdida de derechos y libertades en la Gran Bretaña del Brexit, pasando en otros momentos por las reminiscencias de la infancia de ella, así como por la nostalgia recurrente de Daniel por su hermana muerta y por la olvidada pintora Pauline Boty; encuadrados en el marco del otoño que se cierne sobre ellos, dejando sin embargo briznas de color desde las que remontar el entorno desolado que les atenaza. Procedo a examinar el abanico de facetas que se va modulando a lo largo de la narración, culminando en la melancolía moderna, que postula Bartra, y en la consideración de la transcripción con la tinta de la melancolía que, como dice Starobinski, transmuta la negrura en luz.
\end{abstract}

PALABRAS CLAVE: Ali Smith, Bachelard, Pauline Boty, Brexit, melancolia, nostalgia, écfrasis.

\section{ASPECTS OF MELANCHOLY IN THE NOVEL AUTUMN BY ALI SMITH}

ABSTRACT: Ali Smith is a great creator of melancholic atmospheres. In her novel Autumn we are submerged in the nostalgia of life by a hundred years old man, Daniel. A nostalgia that acquires political aspects in the young Elisabeth, who is facing the loss of rights and liberties in the Brexit Great Britain. We then reflect on her childhood memories and Daniel's recurrent nostalgia for his dead sister and the forgotten painter Pauline Boty. These situations are framed by the autumn that hangs over them, but allows the emergence of shades of colour from which the desolate 
environment can be surmounted. I examine this kaleidoscope of melancholic aspects, which concludes with the modern melancholy, posited by Bartra, and the consideration of the transcription with the ink of melancholy that, as Starobinski says, transforms darkness into light.

KEYWORDS: Ali Smith, Bachelard, Pauline Boty, Brexit, melancholy, nostalgia, ekphrasis.

\title{
ASPECTS DE LA MÉLANCOLIE DANS LE ROMAN AUTOMNE D'ALI SMITH
}

\begin{abstract}
RÉSUMÉ : Ali Smith est une grande créatrice d'atmosphères mélancoliques. Dans son roman Automne, elle nous plonge dans la nostalgie de vivre éprouvée par un homme centenaire, Daniel. Cette nostalgie prend des teintes politiques lorsque la jeune Elisabeth est confrontée à la perte des droits et des libertés dans la Grande Bretagne du Brexit. Nous traversons aussi les réminiscences de son enfance et la nostalgie récurrente ressentie par Daniel pour la perte de sa sœur et de Pauline Boty, peintre oubliée. L'ensemble a pour cadre l'automne qui plane sur eux libérant cependant quelques fils de couleur, qui permettent de surmonter l'environnement désolé qui les tourmente. Je procède à examiner la palette de facettes qui peu à peu se modèle tout au long du récit et qui culmine avec la mélancolie moderne qu'énonce Bartra et avec la considération de la transcription dans l'encre de la mélancolie qui comme dit Starobinski transmute l'obscurité en lumière.
\end{abstract}

MOTS CLÉS : Ali Smith, Bachelard, Pauline Boty, Brexit, mélancolie, nostalgie, ekphrasis.

Recibido: 16/02/2020. Aceptado: 21/09/2020

\section{Introducción}

Ali Smith es una escritora británica, nacida en 1962 en Inverness (Escocia). Ha sido profesora universitaria de literatura inglesa, escocesa y americana en Glasgow, y vive actualmente en Cambridge. En su faceta literaria ha publicado hasta el momento, entre otros textos, siete obras de teatro ${ }^{1}$, cinco colecciones de

1. Stalemate, The Dance, Trace of Arc, Amazons, Comic, The Seer, Just. 
cuentos $^{2}$, un libro para niños ${ }^{3}$, un relato autobiográfico ${ }^{4}$ y nueve novelas ${ }^{5}$, por las que ha recibido numerosos premios y nominaciones. Asimismo, colabora habitualmente en prensa, con artículos para The Guardian, de Scotsman y otros periódicos. En sus obras cobran especial importancia aspectos tales como el poder de la literatura, el paso del tiempo, las tensiones sociales, la esperanza y la belleza.

En Autumn 'Otoño', publicada en 2016, nos sumerge en una atmósfera melancólica al lograr que sus personajes vivan su difícil presente entreverado con las memorias luminosas del pasado, suscitando un sentimiento complejo, mezcla de tristeza y gozo, que va modulando en diferentes facetas a lo largo de la trama.

Me baso para la clasificación de estas facetas de la melancolía en una tipología establecida por mí misma y extraída de la propia lectura de la novela que nos ocupa: nostalgia de la juventud, conciencia de la destrucción por el tiempo, añoranza de la infancia, nostalgia por los seres amados, memoria y añoranza de otra época y melancolía ambiental. Para las dos últimas categorías he tenido en cuenta, respectivamente, los planteamientos efectuados por Roger Bartra (2019), acerca de la melancolía moderna, y por Jean Starobinski (2016: 496-503), así como por Gaston Bachelard $(1965,2011)$-considerado también en otros momentos-, en cuanto a su relación con el espacio y con la imaginación. Entre ellas se echará en falta, no obstante, una de las principales y más frecuentes, que no aparece en el libro dado que los personajes no tienen ocasión de vivirla: la añoranza de los emigrantes por su país

\section{Nostalgia de la juventud}

La novela, narrada en tercera persona, alternando estilo indirecto e indirecto libre, comienza describiendo la nostalgia de la juventud. Muestra a este fin el sueño que está teniendo Daniel, un hombre de ciento un años que permanece inconsciente en una residencia de ancianos, La descripción que hace Smith del anciano recuerda vivamente la serie de retratos efectuados en un asilo (cfr. p. ej. The Museum of Modern Art 2018) por el fotógrafo estadounidense Nicholas Nixon, nacido en Detroit, Michigan, en 1947; artista que también tiene series

2. Free Love and Other Stories, Other Stories and Other Stories, The Whole Story and Other Stories, The First Person and Other Stories, Public Library and Other Stories.

3. The Story of Antigone: Save the Story.

4. Shire.

5. Like, Hotel World, The Accidental, Girl Meets Boy, There But For The, Artful, How to Be Both, Autumn, Winter, Spring. 
sobre enfermos de sida o sobre el paso del tiempo, habiendo retratado, por ejemplo, a su mujer y sus cuñadas en una fecha concreta una vez al año desde el verano de 1975 hasta el momento presente -más de cuarenta años en que vamos viendo la evolución de sus rostros- (cfr. galería completa en Minot 2014). En su sueño Daniel, viviendo una ensoñación oceánica como las que describe Bachelard (2011: 259-317), tras identificarse con un árbol se siente atravesando el tiempo hasta una etapa en que su cuerpo era joven, aunque finalmente no puede evitar despertar a la conciencia del presente que le aprisiona en la tristeza:

Daniel Gluck looks from the death to the life, then back to the death again.

The world's sadness.

Definitely still in the world.

He looks down at his leaf coat, still green.

He holds out a forearm, still miraculous, young.

It will not last, the dream.

He takes hold of one leaf at the corner of his coat. He holds it hard. He will take it back with him if he can. Proof of where he's been.

What else can he bring?

How did that chorus go, again?

How many worlds

Handful of sand. (Smith 2016: 13).

Este primer capítulo, en el que una persona semiinconsciente oscila entre el dolor que impregna su situación actual y la libertad del sueño, recuerda el comienzo de la novela de Ursula K. Le Guin The Lathe of Heaven ('El torno del cielo'), a cuyo protagonista, que se percibe a sí mismo como una medusa nadando en un mar sin límites (tal vez reminiscencia de una existencia anterior), sumergido también aquí en una experiencia oceánica como las descritas por Bachelard (ídem), le cuesta abrir los ojos a la realidad que le espera en la vigilia:

Current-borne, wave-flung, tugged hugely by the whole might of ocean, the jellyfish drifts in the tidal abyss. The light shines through it, and the dark enters it. Borne, flung, tugged from anywhere to anywhere, for in the deep sea there is no compass but nearer and farther, higher and lower, the jellyfish hangs and sways [...] And now, now the currents mislead and the waves betray, breaking their endless circle, to leap up in loud foam against rock and air, breaking... What will the creature made all of seadrift do on the dry sand of daylight; what will the mind do, each morning, waking? (Le Guin 2008: 1-2). 
Seguidamente aparece en la narración Elisabeth, de treinta y dos años, profesora eventual de Historia del Arte en una Universidad de Londres que, por falta de ingresos, ha tenido que volver a vivir con su madre y lucha con la burocracia para renovar su pasaporte. Sin embargo, por muchas que sean sus ocupaciones, todos los días va a ver a Daniel y, aunque no es seguro que pueda oírla, le lee fragmentos de obras clásicas de la literatura universal (Historia de dos ciudades de Dickens; las Metamorfosis de Ovidio; Un mundo feliz de Huxley), confiando en que sean tan catárticas para él como lo son para ella. En la residencia creen que es su nieta, pero en realidad fueron vecinos que compartieron infinidad de horas durante la infancia de ella, cuando su madre la dejaba al cuidado de Daniel, quien, siendo compositor y estando ya retirado, disponía de mucho tiempo libre.

\section{Conciencia de la destrucción por el tiempo}

La novela introduce luego la conciencia de la destrucción por el tiempo. A este fin intercala los sueños y pensamientos de Daniel con el presente y el pasado de Elisabeth y su madre, las cuales viven sumergidas en las tensiones de la Inglaterra del Brexit y los problemas de integración social propiciados por la crisis.

En uno de los últimos capítulos, aparece también Pauline Boty, una olvidada actriz y pintora pop art londinense, que vivió entre 1938 y 1966 y sobre la que versa la tesis de Elisabeth, quien había quedado sorprendida al descubrirla en un viejo catálogo en una tienda de arte y darse cuenta de que ya conocía sus cuadros, por habérselos descrito minuciosamente Daniel, el cual los había visto crear y estaba enamorado de la mirada vital de la pintora. Algunas de estas obras (cfr. una selección en WikiArt.org: s/f), que Smith presenta profusamente ante el lector en un magnífico ejercicio de écfrasis ${ }^{6}$, son: Colour Her Gone, autorretrato; The Only Blonde in the World, retrato de Marilyn Monroe; It's A Man's World I y $I I$, dos cuadros en los que denunciaba los estereotipos vigentes en el siglo XX por los que se tendía a identificar exclusivamente a las mujeres con su cuerpo y a

6. Utilizo aquí el término écfrasis en el doble sentido que le da Rifatterre: tanto la representación de otro arte con palabras, como una descripción especialmente vívida:

Entiendo pues écfrasis en el sentido que tiene la palabra para la teoría literaria, en la que designa un caso particular de descripción o de relato que dio origen a un género menor cuyos procedimientos son del orden de la mímesis [...] esta acepción moderna no es en absoluto incompatible con el sentido que los antiguos daban a la écfrasis, considerada como un tropo sinónimo de la hipotiposis -descripción tan llamativa y sugestiva que uno se creería en presencia del objeto mismo. (2000: 161). 
los hombres con su trabajo; Bum, que pintó poco antes de morir, estando ya muy enferma, y en el que -como si diese un corte de mangas a la muerte- muestra su sentido del humor; y el retrato de Christine Keller, una cantante y presunta espía soviética en Reino Unido, cuya detención dio lugar a un gran escándalo, el caso Profumo; cuadro que aparece en una foto de la época pero que ha desaparecido, lo cual da lugar a una reflexión melancólica por parte de Elisabeth acerca de las obras perdidas de Boty, tal vez arrumbadas en un pajar y pasto de una lenta destrucción.

\section{Añoranza de la infancia}

Paralelamente, la narración va revelando el peso anímico de la añoranza de la infancia, ponderado por Bachelard como componente esencial del ánima (2011: 149-217). A lo largo de los capítulos, vamos descubriendo la profundidad de los sentimientos de Elisabeth hacia Daniel, por el que siente una gran admiración y amor platónico. En los recuerdos de sus encuentros de la época infantil captamos la nostalgia por el tiempo que compartieron, en el que creaban historias a partir de imágenes e imágenes a partir de historias o discutían sobre su verdad, la verdad de las historias, o sobre las emociones que transmitían las pinturas, o iban al teatro a ver La Tempestad de Shakespeare o a pasear por el campo, aunque a él ya entonces le costaba un poco moverse.

\section{Nostalgia por los seres amados}

A esa añoranza por un tiempo desaparecido se une la nostalgia por los seres amados. En los pensamientos de Daniel, que surgen a través del sueño en que se halla sumido, brota no solo la melancolía por la juventud perdida sino la nostalgia, que nunca le abandona, por su hermana, que murió trágicamente durante el nazismo y por la que sentía una gran pasión no correspondida; nostalgia que se funde con la que le produce el recuerdo de su amor platónico por la pintora Pauline Boty, bajo cuya ventana fue un día a cantar su tristeza identificándose con Michael Furey, el enamorado que la protagonista de Los muertos de Joyce no podía olvidar tras haberle escuchado entonar bajo la lluvia The Last of Aughrim y saber que por ir a despedirse de ella había muerto de tisis, a consecuencia de haberse mojado hasta los tuétanos (cfr. Joyce 1977: 207-213). Pauline también murió poco tiempo después, al dar a luz, tras un cáncer del que no quiso tratarse para no perjudicar al feto. 


\section{Memoria y añoranza de otros tiempos}

La memoria y añoranza de otros tiempos se intensifica a través del retrato que hace Smith de la madre de Elisabeth, Wendy, cuya mirada melancólica abarca morosamente los objetos del pasado reunidos para una subasta y los programas de televisión de antaño. Wendy acaba, sin embargo, rescatando de aquel tiempo la presencia real de Zoe, una actriz a la que admiraba entonces y que ahora, inesperadamente, pasa a formar parte de su vida, convirtiéndose en su pareja.

Tanto Wendy como Elisabeth y Zoe añoran tiempos mejores del Reino Unido anteriores al Brexit, donde había más solidaridad con los refugiados y condiciones sociales más soportables para todos. Wendy lleva su reivindicación a tirar objetos contra una verja opresiva, ante la que anteriormente Elisabeth había reflexionado melancólicamente sobre las barreras físicas y psicológicas que están imponiéndose en las sociedades occidentales. Ambas generaciones de mujeres se hallan inmersas en la pérdida generalizada de esperanza de la que, como señala Roger Bartra en La melancolía moderna, está imbuida nuestra época:

\footnotetext{
Ocurre a veces que la corriente llega a depresiones donde el agua se estanca. Es el río del humor negro, de la melancolía hipocrática, del spleen y de la acedia. Sus aguas impregnan la sociedad moderna de flujos intrigantes. Es un misterio la presencia generalizada de tristezas, tedios, melancolías y locuras, todas ellas expresiones que contradicen las fuerzas dominantes de la modernidad, que tienden a establecer la hegemonía de la eficiencia, la claridad y la racionalidad. [...] Son una señal de que el mundo está entrando en una nueva época, dominada por un extraño capitalismo tardío cuyos signos apenas estamos comenzando a descifrar. (Bartra 2019: 9-10).
}

Wendy y Zoe añoran también una época de menor corrección política, en la que cuadros como los de Boty, en los que aparecen con naturalidad cuerpos desnudos, podían ser mostrados sin escándalo.

\section{Melancolía ambiental}

Por otra parte, a lo largo del texto subyace una melancolía ambiental, pues como marco de todas estas historias hilvanadas de tristeza está el otoño, la estación que se cierne sobre los personajes dejando sin embargo briznas de color desde las que remontar la desolación que les atenaza. 
Tanto Starobinski (2016: 496-503) como Bachelard $(1965,2011)$ aluden al espacio como fuente de melancolía. En este caso se trata de un lugar empapado por el frío atmosférico. Sin embargo, la literatura puede operar sobre él al transformarlo en palabras y hallar un contrapunto en otros elementos del paisaje, como una solitaria rosa o su huella, evocando tenazmente la intimidad de la casa y la fragilidad del nido.

De esta manera, al igual que Antonio Machado, en Campos de Castilla, veía el retorno de la esperanza en la rama brotada de un olmo seco:

\author{
Olmo, quiero anotar en mi cartera \\ la gracia de tu rama verdecida. \\ Mi corazón espera \\ también, hacia la luz y hacia la vida, \\ otro milagro de la primavera. (1977: 121-122),
}

\title{
Ali Smith termina Otoño con estas palabras:
}

But there are roses, there are still roses.

In the damp and the cold, on a bush that looks

done, there's a wide-open rose, still.

Look at the colour of it. (2016: 259-260).

Ya en una obra anterior, el relato May 'Mayo' (2003: 53-69), la autora exaltaba otros colores, el blanco y el verde, con esta misma función de ancla para la esperanza en medio de un paisaje desolado:

\footnotetext{
Then I thought about the tree. It was the most beautiful tree I have ever seen. [...] It was bluesky white, heat-gaze white, the white of the sheets that you bring in from the line in the garden dry after hardly any time because the air is so warm. It was the white of sun, the white that's behind all the colours there are, it was open-mouthed white on open-mouthed white, swathes of sweet-smelling outheld white lifting and falling and nodding, saying the one word yes over and over, white spilling over itself. It was a white that longed for bees, that wanted you inside it, dusted, pollen-smudged; it was all the more beautiful for being so brief, so on the point of gone, about to be nudged off by the wind and the coming leaves. It was the white before green, and the green of this tree, I knew, would be even more beautiful than the white; I knew that if I were to see it in leaf I would smell and hear nothing but green. (2003: 55-56).
} 
Análogamente, en su siguiente novela, Winter 'Invierno', no es un color sino la huella de una flor que había estado insertada en un libro la que despierta en el protagonista un sentimiento de belleza que le permite remontar la opresión causada por las pérdidas sociales y personales:

The ghost of a flower is more what it looks like. Who knows who pressed it in the book, who knows when? There it is. The shape left by the bud makes it like the ghost of a flame too, like the shadow of a steady little flame. [...] It's the ghost of a flower not yet open on its stem, the real thing long gone, but look, still there, the mark of the life of it reaching across the words on the page for all the world like a footpath that leads to the lit tip of a candle. (2017: 319).

Sin embargo, tales epifanías, centradas en una miniatura significativa (cfr. Bachelard 1965: 140-142), no serían posibles sin el contraste radiante de la atmósfera melancólica en que surgen, pues como apunta en La tinta de la melancolía Jean Starobinski señalando el papel que esta juega al transformarse en palabra poética: "La melancolía, transmutada en tinta, se convierte a su vez en el azogue gracias al cual la imagen resplandece. La oscuridad más densa pone frente a la luz una superficie donde ella reverbera, luciferina, como si emanara de una segunda fuente" (2016: 503).

\section{Conclusión}

De este modo, como en obras anteriores y posteriores suyas, Ali Smith al final de Otoño, tras haber recorrido con sus protagonistas diferentes facetas de la melancolía (nostalgia de la juventud, conciencia de la destrucción por el tiempo, añoranza de la infancia, nostalgia por los seres amados, memoria y añoranza de otra época, melancolía ambiental), y habernos llevado, como consecuencia, a ser plenamente conscientes de las pérdidas ocasionadas tanto por el paso ineludible del tiempo y de la vida como por las decisiones políticas que han modificado nuestro entorno social, nos incita a respirar confortados ante la descripción de esa rosa rebosante de color que, símbolo de una irrenunciable esperanza, se brinda a nuestra consideración en su esplendorosa plenitud; como he observado en este trabajo, con ayuda de Bachelard, Starobinski y Bartra. 


\section{Bibliografía}

BACHELARD, G. (1965 [1957]). La poética del espacio. México: Fondo de Cultura Económica. Trad. E. de Champurcín.

BACHELARD, G. (2011 [1960]). La poética de la ensoñación. México. Fondo de Cultura Económica. Trad. I. Vitale.

BARTRA, R. (2019 [2017]). La melancolía moderna. Valencia: Pre-Textos.

DICKENS, Ch. (2006 [1859]). Historia de dos ciudades. Madrid: Cátedra. Ed. P. Hidalgo. Trad. J. J. Zaro.

HUXLEY, A. (2013 [1932]). Un mundo feliz. Madrid: Cátedra. Ed. y trad. J. I. Gómez López.

JOYCE, J. (1977 [1914]). "Los muertos". Dublineses. Barcelona: Círculo de Lectores. Trad. G. Cabrera Infante. 167-214.

LE GUIN, U. K. (2008 [1971]). The Lathe of Heaven. New York: Scribner.

MACHADO, A. (1977 [1912]). Campos de Castilla. Madrid: Taurus.

MINOT, S. (2014). "Forty Portraits in Forty Years: Photographs by Nicholas Nixon". The New York Times Magazine. 3 oct. <https://www.nytimes.com/ interactive/2014/10/03/magazine/01-brown-sisters-forty-years.html>.

OVIDIO (2005 [8 d.C.]). Metamorfosis. Madrid, Alianza Editorial. Trad. A. Ramírez Verger.

RIFFATERRE, M. (2000 [1994]). "La ilusión de écfrasis". Literatura y pintura. Madrid: Arco Libros. Ed. Antonio Monegal. Trad. C. Besa. 161-183.

SHAKESPEARE, W. (2016 [1612]). La tempestad. Madrid: Alianza Editorial. Trad. M. Astrana Marín.

SMITH, A. (1986). Stalemate. Edinburgh: E. Festival Fringe.

SMITH, A. (1988). The Dance. Edinburgh: E. Festival Fringe.

SMITH, A. (1989). Trace of Arc. Edinburgh: E. Festival Fringe.

SMITH, A. $\left(1990^{1}\right)$. Amazons. Cambridge: Footlights.

SMITH, A. $\left(1990^{2}\right)$. Comic. Edinburgh: E. Festival Fringe.

SMITH, A. (1995). Free Love and Other Stories. London: Virago.

SMITH, A. (1997). Like. New York: Harcourt.

SMITH, A. (1999). Other Stories and Other Stories. London: Penguin Books.

SMITH, A. $\left(2001^{1}\right)$. Hotel World. London: Penguin Books.

SMITH, A. $\left(2001^{2}\right)$. The Seer. London: Faber and Faber.

SMITH, A. (2003). The Whole Story and Other Stories. London: Penguin Books.

SMITH, A. $\left(2005^{1}\right)$. Just. Edinburgh: E. Festival Fringe.

SMITH, A. $\left(2005^{2}\right)$. The Accidental. London: Hamish Hamilton.

SMITH, A. (2007). Girl Meets Boy. Edinburgh: Canongate Books. 
SMITH, A. (2008). The First Person and Other Stories. London: Hamish Hamilton.

SMITH, A. (2011). There But For The. London: Penguin Books.

SMITH, A. (2012). Artful. London: Penguin Books.

SMITH, A. $\left(2013^{1}\right)$. The Story of Antigone (Save the Story). London: Pushkin Children's Books.

SMITH, A. (20132). Shire. North Hykeham: Full Circle Editions.

SMITH, A. (2014). How to Be Both. London: Penguin Books.

SMITH, A. (2015). Public Library and Other Stories. London: Penguin Books.

SMITH, A. (2016). Autumn. London: Penguin Books.

SMITH, A. (2017). Winter. London: Penguin Books.

SMITH, A. (2019). Spring. London: Penguin Books.

STAROBINSKI, J. (2016 [2012]). La tinta de la melancolía. México: Fondo de Cultura Económica. Trad. A. Merlín.

The Museum of Modern Art. (2018). "Nicholas Nixon/C. C./Boston/1983”. MoMA. Features and perspectives on art and culture. Web. $<$ https://www.moma. org $/$ collection/works/47362?artist_id $=4315 \&$ locale $=$ es\&page $=1 \&$ sov referrer $=$ artist $>$.

WikiArt.org. (s/f). "Pauline Boty". WikiArt Enciclopedia de Artes Visuales. Web. $<$ https://www.wikiart.org/es/pauline-boty $>$. 